Supporting Information

\title{
Facile Synthesis of Reductively Degradable Biopolymers Using Cystamine Diisocyanate as a Novel Coupling Agent
}

Xiuxiu Wang, Wentao Lu, Ru Cheng*, Fenghua Meng, Chao Deng, and Zhiyuan Zhong*

${ }^{a}$ Biomedical Polymers Laboratory, and Jiangsu Key Laboratory of Advanced Functional

Polymer Design and Application, College of Chemistry, Chemical Engineering and Materials Science, Soochow University, Suzhou, 215123, P. R. China.

* Corresponding authors. Tel/Fax: +86-512-65880098, E-mail: rcheng@ suda.edu.cn (R. Cheng); zyzhong@suda.edu.cn (Z. Zhong) 


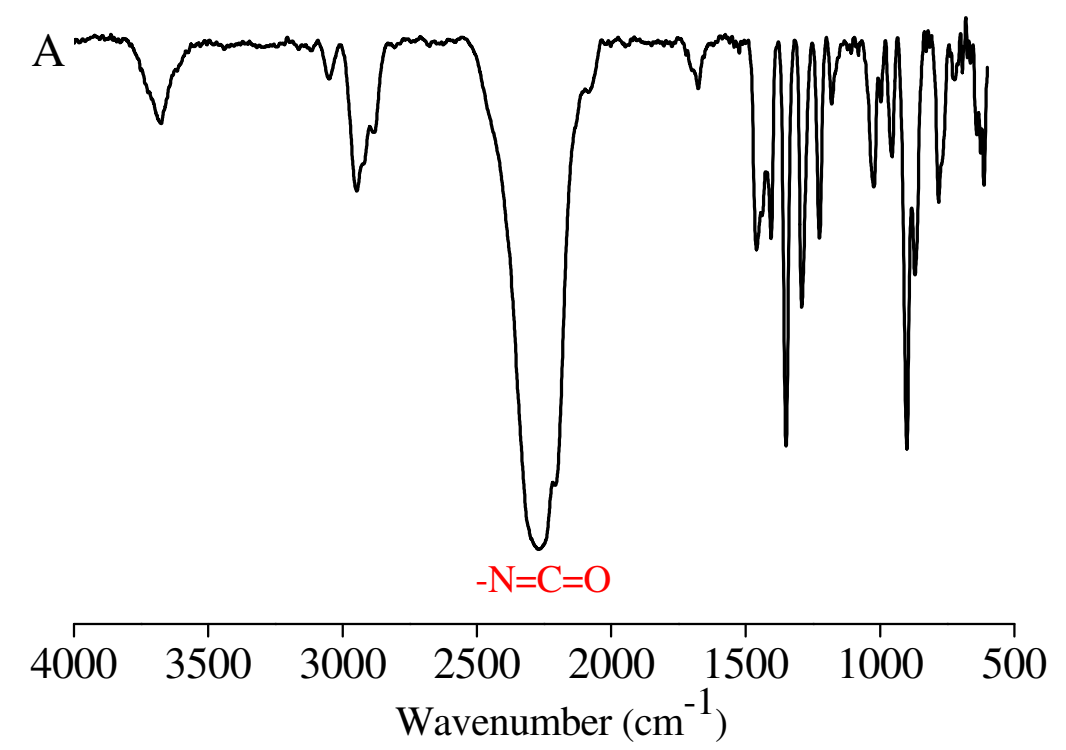

B<smiles>O=CNCCSSCCCN=O</smiles>

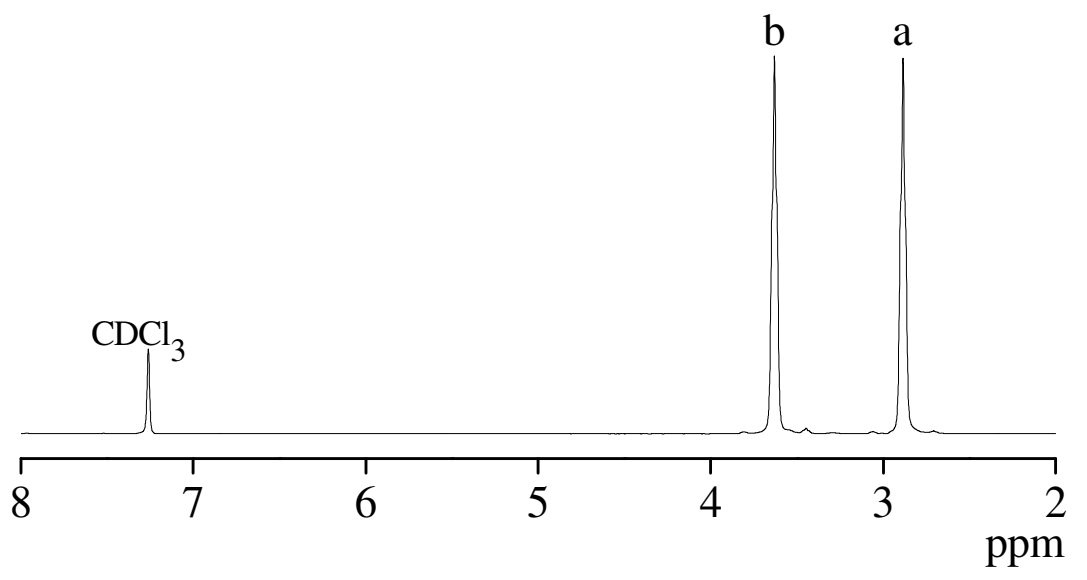


C
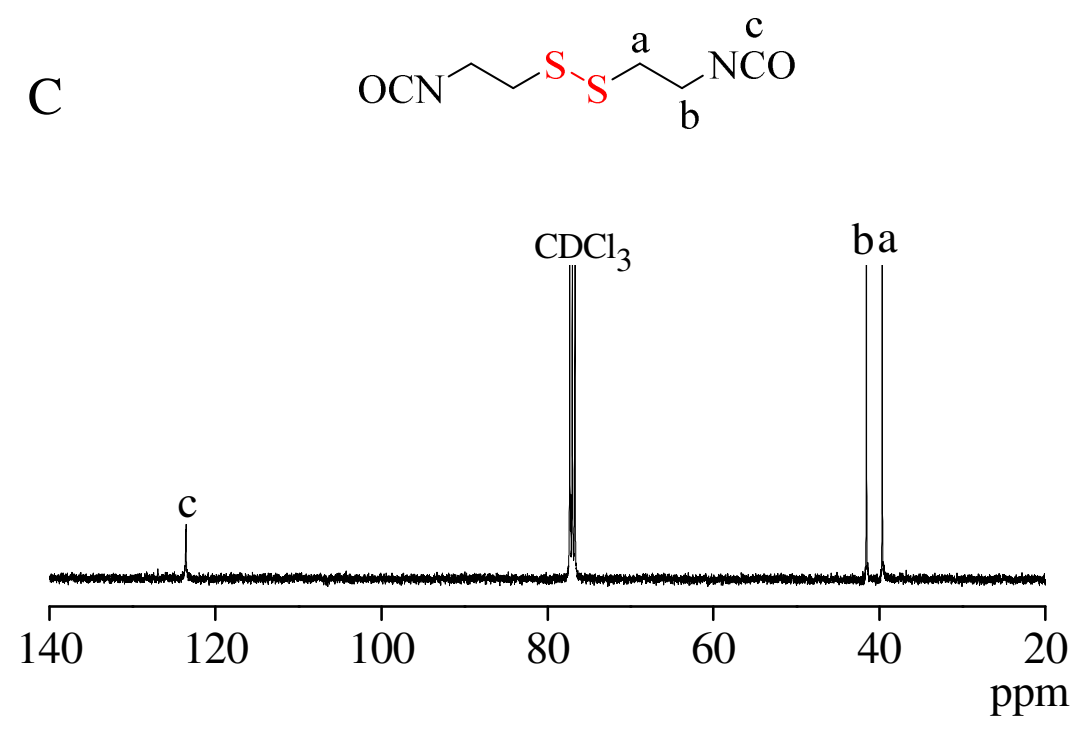

Figure S1. FT-IR (A), ${ }^{1} \mathrm{H}$ NMR $\left(400 \mathrm{MHz}, \mathrm{CDCl}_{3}\right)(\mathrm{B})$, and ${ }^{13} \mathrm{C} \mathrm{NMR}\left(150 \mathrm{MHz}, \mathrm{CDCl}_{3}\right)$ spectra (C) of CDI.

$$
\mathrm{H}_{2} \mathrm{~N}_{\mathrm{b}}^{\mathrm{a}} \mathrm{S}^{-} \mathrm{S}_{\mathrm{a}}^{\mathrm{b}} \mathrm{NH}_{2}
$$

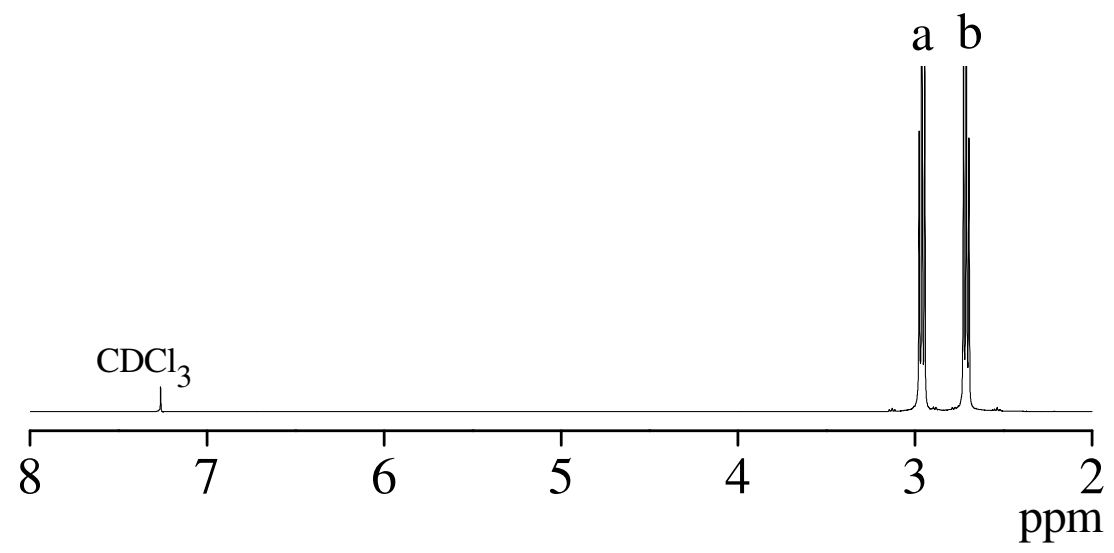

Figure S2. ${ }^{1} \mathrm{H}$ NMR spectrum $\left(400 \mathrm{MHz}, \mathrm{CDCl}_{3}\right)$ of $\mathrm{CDH}$. 


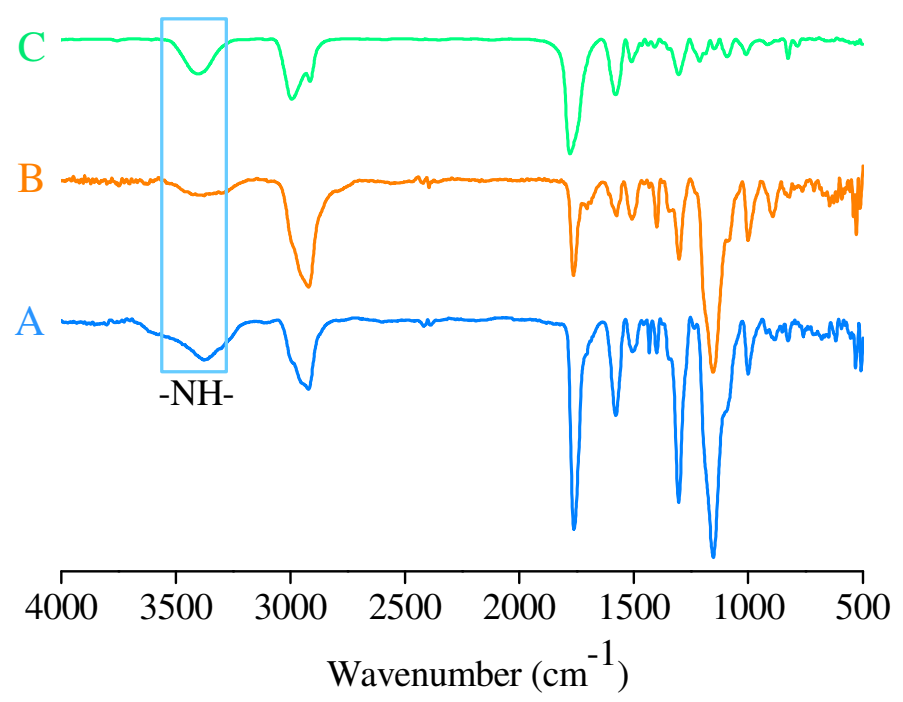

Figure S3. FT-IR spectra of $\operatorname{SSPEG}_{1.5 \mathrm{k}}(\mathrm{A}), \mathrm{SSPEG}_{0.4 \mathrm{k}}(\mathrm{B})$ and $\operatorname{SSPCL}_{0.53 \mathrm{k}}(\mathrm{C})$.
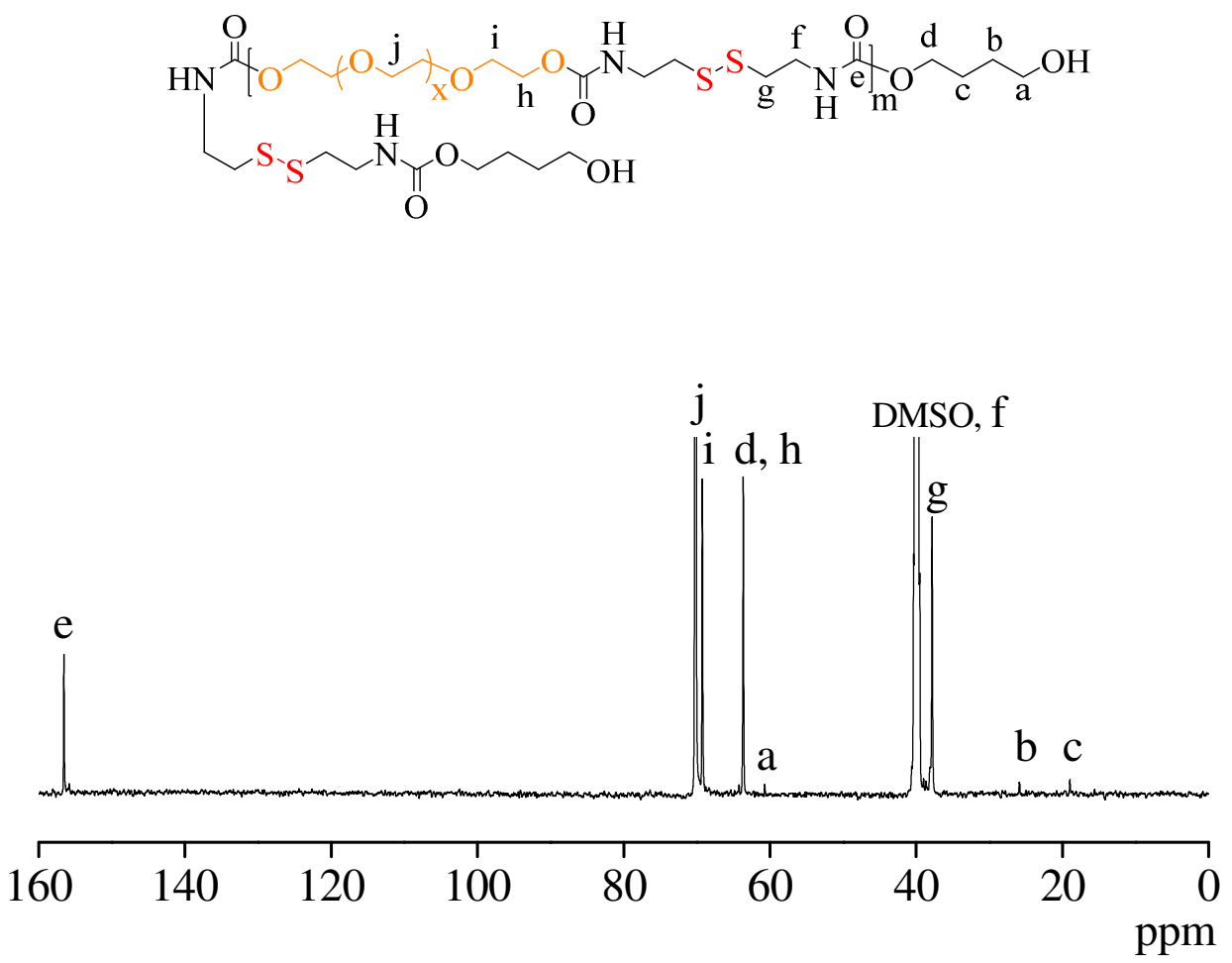

Figure S4. ${ }^{13} \mathrm{C}$ NMR spectrum (150 MHz, DMSO- $\left.d_{6}\right)$ of SSPEG (Table 1, Entry 4). 

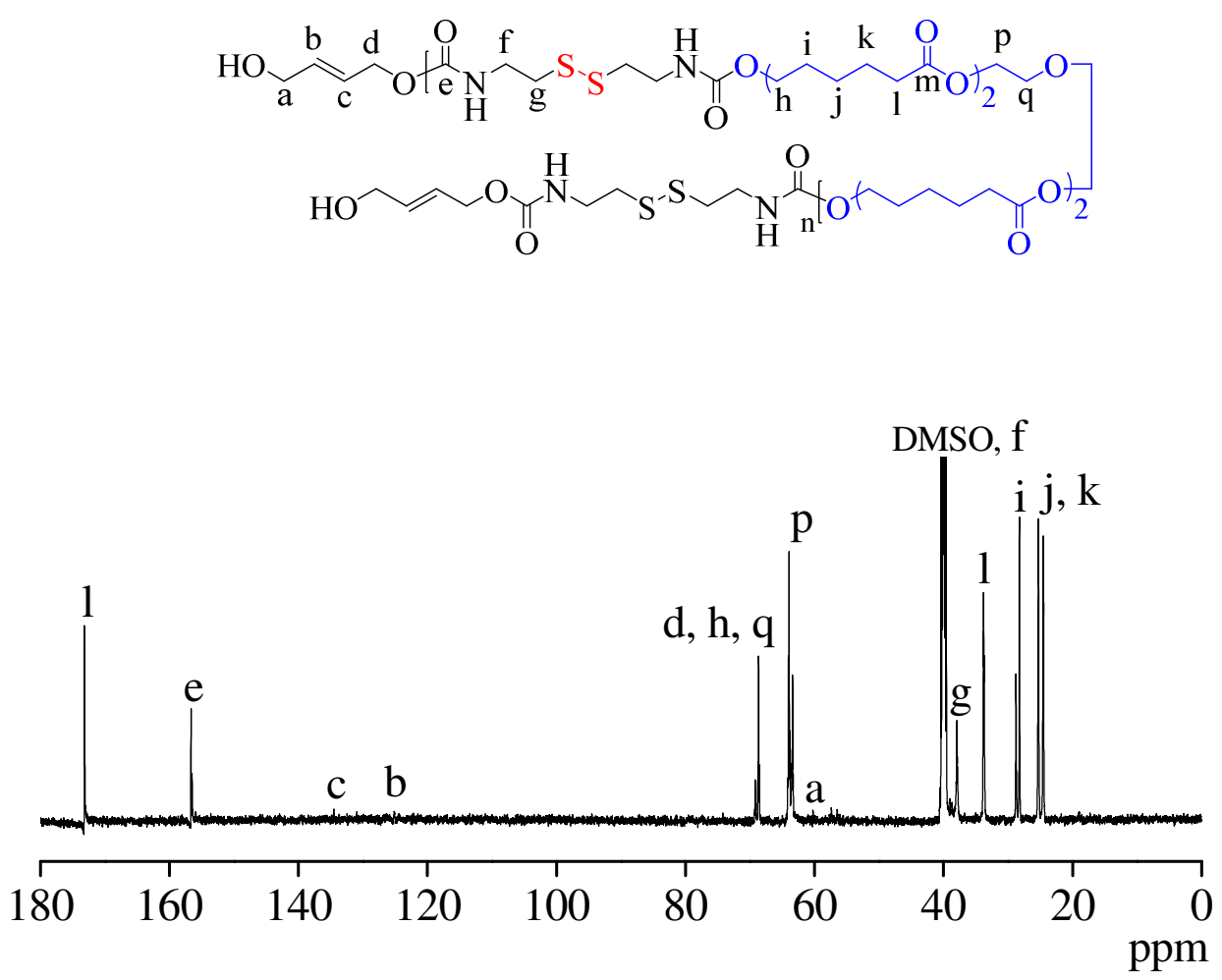

Figure S5. ${ }^{13} \mathrm{C}$ NMR spectrum $\left(150 \mathrm{MHz}\right.$, DMSO- $\left.d_{6}\right)$ of SSPCL (Table 2, Entry 1).

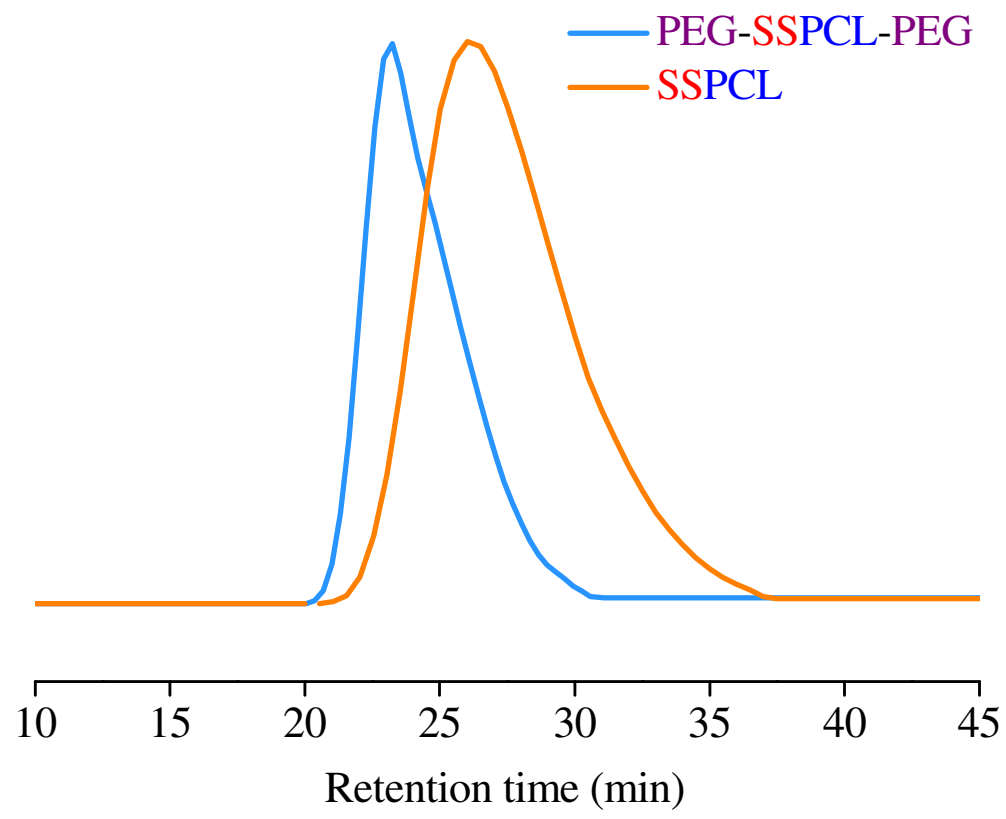

Figure S6. GPC chromatograms of SSPCL (Table 2, Entry 1) and PEG-SSPCL-PEG triblock copolymer. 


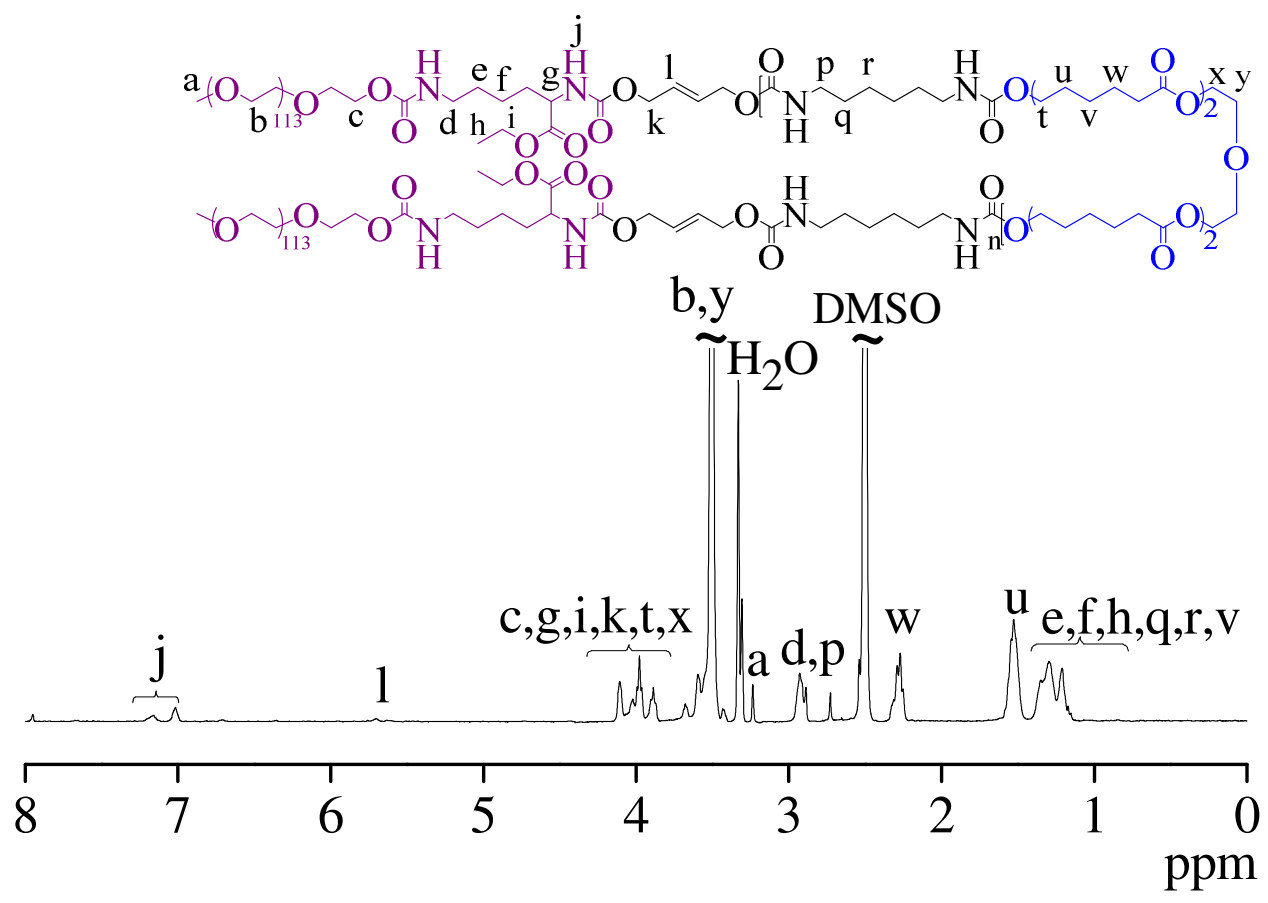

Figure S7. ${ }^{1} \mathrm{H}$ NMR spectrum $\left(400 \mathrm{MHz}, \mathrm{DMSO}-d_{6}\right)$ of PEG-PCL(HDI)-PEG.

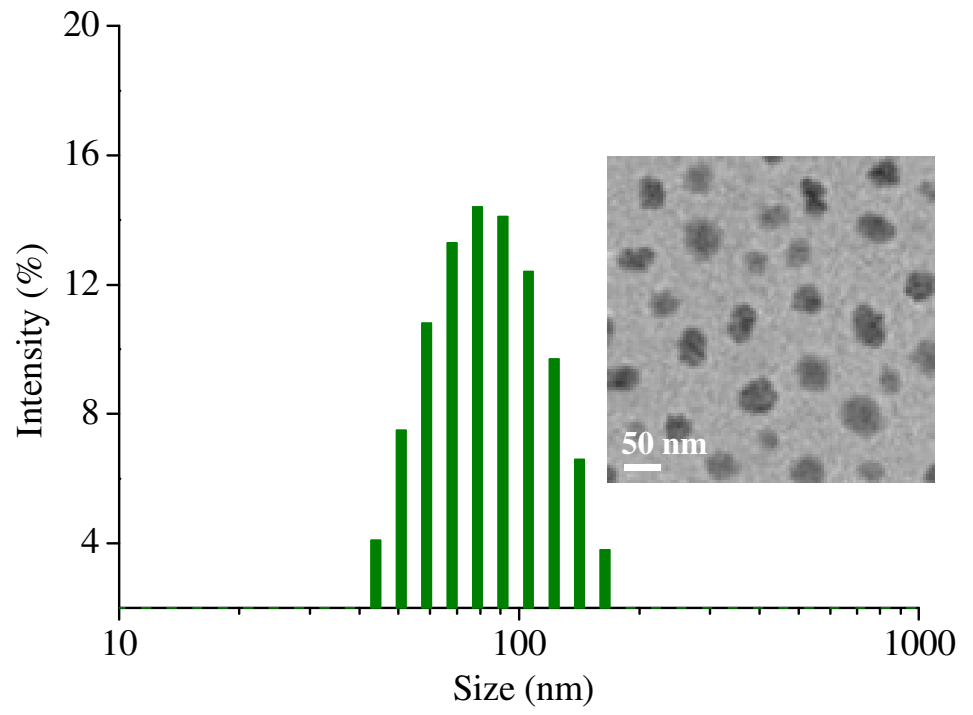

Figure S8. Size distribution of PEG-PCL(HDI)-PEG micelles determined by DLS and TEM. 